\title{
Time course of neuro-mechanical changes underlying stretch-shortening cycle during intermittent exhaustive rebound exercise
}

\author{
Cédric Morio · Pascale Chavet $\cdot$ Philippe Androuet \\ Matthieu Foissac · Eric Berton · Caroline Nicol
}

Received: 8 October 2010 / Accepted: 29 January 2011 / Published online: 19 February 2011

(C) Springer-Verlag 2011

\begin{abstract}
This study analysed the time course of neuromechanical changes underlying stretch-shortening cycle during intermittent exhaustive rebound exercise. On a sledge apparatus, ten subjects repeated until exhaustion a series of 30 unilateral submaximal rebounds, with intermediate 3-min rest periods. Rebound height, ground reaction force, 3D tibial acceleration and electromyographic activity of major lower limb muscles were recorded. A maximal drop jump test performed before and after the exhaustive exercise revealed a $10 \%$ drop in maximal stretch-shortening cycle (SSC) performance. Specific investigation of the neuro-mechanical changes along the exhaustive exercise included classical comparison of the first (BEG) and last (END) rebound series. From the initial accommodation phase, an optimized (OPTIM) series was individually determined as the first of at least two subsequent series with significantly shorter contact time than in the BEG series. The OPTIM series was reached after $3 \pm 1$ series, with associated increased lower limb stiffness during the braking phase and decreased muscle activities during the push-off. The major result was that the early (BEG-OPTIM) changes explained most of the BEG-END ones whereas the actual (OPTIM-END) fatigue effects remained quite limited. This confirmed our expectation that erroneous quantification of
\end{abstract}

Communicated by Jean-René Lacour.

C. Morio $(\bowtie) \cdot$ M. Foissac

Oxylane Research, 4 boulevard de Mons,

BP 299, 59650 Villeneuve d'Ascq Cedex, France

e-mail: cedric.morio@ hotmail.fr

C. Morio - P. Chavet $\cdot$ P. Androuet · E. Berton · C. Nicol

UMR 6233, Institut des Sciences du Mouvement,

Université de la Méditerranée, Marseille, France the SSC fatigue effects might be drawn when using the early beginning of rebound exercise on the sledge as a reference. Actual fatigue effects included medio-lateral instability as suggested by increased peroneus longus preactivation and medio-lateral tibial acceleration. The present methodology is thus considered as improving the distinction between SSC optimization and its deterioration with fatigue.

Keywords Stretch-shortening cycle - Optimization . Fatigue · Tibial acceleration · Lower limb stiffness · Wavelet analysis

\section{Introduction}

Stretch-shortening cycle (SSC) defines natural forms of locomotion such as running and hopping, and it is considered as providing unique possibilities to study normal and fatigued muscle function (Komi 2000; Komi and Nicol 2010). Examination of the neuro-mechanical adjustments to SSC task is favoured by the facts that this type of exercise involves a large number of muscles, different forms of muscle actions as well as combined interventions of central and reflex neural components. In prolonged SSC exercises, however, one of the critical features consists in revealing the early signs of fatigue to quantify and understand its progressive development. In this context, fatigue is considered as "any exercise-induced reduction in the ability to exert muscle force or power regardless of whether the task can be sustained or not" (Bigland-Ritchie and Woods 1984). Accordingly, the inability to continue a task, often termed "exhaustion", is considered as the culmination of these ongoing fatigue processes (for a review, see Enoka and Duchateau 2008). 
Due to the multiplicity of the neural strategies potentially involved in submaximal SSC exercise, the protocols are commonly including a warm-up and a practice period before the actual exercise. In most running fatigue studies, the practice is reported as unmonitored and lasting 3-6 min to allow sufficient time to reach a stable oxygen uptake and/ or some accommodation to the task (e.g. Candau et al. 1998; Avogadro et al. 2003). Fatigue is then examined from the remaining exercise period. This differs from several biomechanical running and jumping studies in which the early beginning of exercise after warm-up is usually taken as the reference for pre-post fatigue comparison (Nicol et al. 1996; Verbitsky et al. 1998; Hardin et al. 2004). As demonstrated, however, by the $10-\mathrm{km}$ running study of Finni et al. (2003) the greatest neuro-mechanical changes are very likely to place during the early kilometres of the run. In rebound fatigue studies more emphasis has been put on the progressive neuro-mechanical adjustments that are taking place during the exercise. In hopping, although Bonnard et al. (1994) did not examine the first 2 min of the exercise, the accent was put on the intra- and inter-joint neuromuscular adaptations to fatigue. In a continuous SSC rebound exercise on a sledge apparatus, Horita (2000) reported the initial third of the exercise duration (1-2 $\mathrm{min}$ ) to be characterised by an overall reduction in muscle preactivation, with large opposite trends of EMG changes in the quadriceps and triceps surae muscle groups. In an intermittent SSC rebound exercise, Regueme et al. (2005a) reported large reductions in soleus and gastrocnemius muscle preactivation as well as in contact time to occur during the early $25 \%$ of the exhaustive exercise duration. Independently of the continuous versus intermittent form of the rebound exercise and despite the preliminary use of a practice period or session, these studies reported in all subjects the need for tens of rebounds to get a so-called "optimized" SSC pattern. This is therefore questioning the actual meaning of some of our previous pre-post fatigue observations after exhaustive rebound exercise, such as a large $(44 \%)$ reduction in the lateral gastrocnemius preactivation (Nicol et al. 1996). Additional data are needed, however, to re-examine in particular the intra- and inter-limb strategies that might reflect either SSC optimization or fatigue compensation during the time course of the exercise.

In the optimized state, it is suggested that a proper preactivation of the lower limb muscles before touch down and their elevated activation during the braking phase allow the muscle-tendon complex to damp the impact to resist the imposed stretch and, thus, to store elastic energy. This energy may then be recoiled with a short time delay during push-off (Komi 2000). The concept of elastic storage supports the existence of reflex activation since high muscular activation during the braking phase of SSC is a prerequisite for efficient storage of elastic energy (Komi and Gollhofer
1997). Coactivation of bi-articular leg muscles during stance phase is also reported as clearly linked to running economy through greater elastic energy return (Heise et al. 2008). In this line, leg stiffness is considered as improving the use of the tendon lengthening during the braking phase (Spurrs et al. 2003; Cormie et al. 2010) and, thus, as contributing to SSC performance enhancement (Cormie et al. 2010) and to the low cost of running (Dalleau et al. 1998; Spurrs et al. 2003; Clark 2009). In this line, well-trained jumpers (Kyröläinen and Komi 1995a, b) and distance runners (Paavolainen et al. 1999) are reported with higher pre-activation and EMG ratio between the eccentric and concentric phases than athletes of lower level. Short contact times are also demonstrated to be required at both economical running and high top running speed (Nummela et al. 2007). According to Hasegawa et al. (2007), both shorter contact time and inversion at the foot contact might contribute to higher running economy. Emphasizing the use of the contact time in SSC performance quantification, Flanagan and Comyns (2008) evaluated jumping performances by the reactive strength index (RSI), calculated as the rebound height divided by the contact time, in which an increased RSI results from a higher rebound and/or a shorter contact time.

As a logical consequence of these observations, several fatigue running and rebound studies reported a loss of tolerance to ground impact, leading to longer braking and total contact time with associated increased work during the push-off phase (Gollhofer et al. 1987; Nicol et al. 1991; Horita 2000; Kuitunen et al. 2007). This may be considered as a vicious circle that would result in progressively impaired capacity to maintain the task (Nicol and Komi 2010) and in increased shock transmitted to the tibia (Mizrahi et al. 2000). These trends differed from the "safer style" adopted during prolonged running (Nummela et al. 2008; Morin et al. 2009; Martin et al. 2010), but in these racing type conditions the subjects could freely adjust their running pace. When considering the neuro-mechanical fatigue effects reported at constant submaximal running speed or rebound height, only a few studies have been performed that found either no significant change in EMG (Paavolainen et al. 1999) or different and limited neural strategies (Nicol et al. 1996; Mizrahi et al. 2000; Regueme et al. 2005a). The latter two running and rebound studies (Mizrahi et al. 2000; Regueme et al. 2005a) are of particular interest as both of them observed early neural changes along time so that they did not use the beginning of the exercise as the non fatigued reference. Instead, Mizrahi et al. (2000) used the fifth minute of the treadmill run, and Regueme et al. (2005a) used the rebound series performed at $25 \%$ of the individual exercise duration.

Therefore, the present study aimed to analyse the time course of neuro-mechanical changes underlying stretchshortening cycle function during intermittent exhaustive 
rebound exercise. The first hypothesis was that the expected decrease in contact time at the beginning of the SSC rebound exercise could reflect an improved SSC pattern rather than any early neuro-mechanical compensation for fatigue. This raised the question of the actual fatigue and exhaustion effects in such a complex exhaustive SSC exercise. In particular, as the task consisted in reaching a pre-set rebound height, the severity of the shock transmitted through the tibia was expected to increase towards exhaustion.

\section{Materials and methods}

\section{Subjects}

Ten healthy subjects ( 9 males and 1 female; $25 \pm 3$ years; $71 \pm 8 \mathrm{~kg} ; 1.74 \pm 0.06 \mathrm{~m}$ ) volunteered to participate in this study. They were free from lower-limb injury and all of them provided written informed consent. The study was conducted according to the Declaration of Helsinki (2002) and approved by the Local Committee for Human Protection in Biomedical Research (visa $\mathrm{N}^{\circ} 04$ 008).

\section{Protocol}

As shown in the upper graph of Fig. 1, the experimental design included an intermittent exhaustive SSC (rebound) exercise performed with the dominant lower limb in a sitting position on a sledge apparatus. To allow sufficient rebounds to be performed before exhaustion, the sledge seat was gliding along rails inclined $23^{\circ}$ from the horizontal (Regueme et al. 2005a). The acute functional fatigue effects were evaluated through maximal and submaximal rebound tests.

\section{Practice period}

The exhaustive rebound exercise was preceded by a standardised 15-min practice that used verbal and visual feedbacks to train the subjects. To ensure that the entire triceps surae muscle group was primarily involved in this exercise as well as in the maximal drop jump (DJ) test, no heel contact was allowed on the sledge force plate and a maximal $90^{\circ}$ knee flexion angle had to be reached during each contact phase (e.g. Regueme et al. 2005a). Based on previous experience, implication of both hip extensors was limited by setting the resting seat inclination at $120^{\circ}$ and by training the subjects to let their knee flex freely between take-off and the subsequent impact. The practice aimed also to determine the dominant rebound lower limb, with its corresponding optimal dropping height producing a maximal rebound height (for details see Nicol et al. 1996). This was followed by a 10-min recovery period that was used to fix all the recording equipments on the subjects.

\section{Intermittent exhaustive rebound exercise}

As shown in Fig. 1, this exercise consisted in $\mathrm{N}$ series of 30 unilateral rebounds performed using the dominant lower limb, with inter-series rest periods of $3 \mathrm{~min}$. The rebound height was individually set at $70 \%$ of the PRE maximal DJ

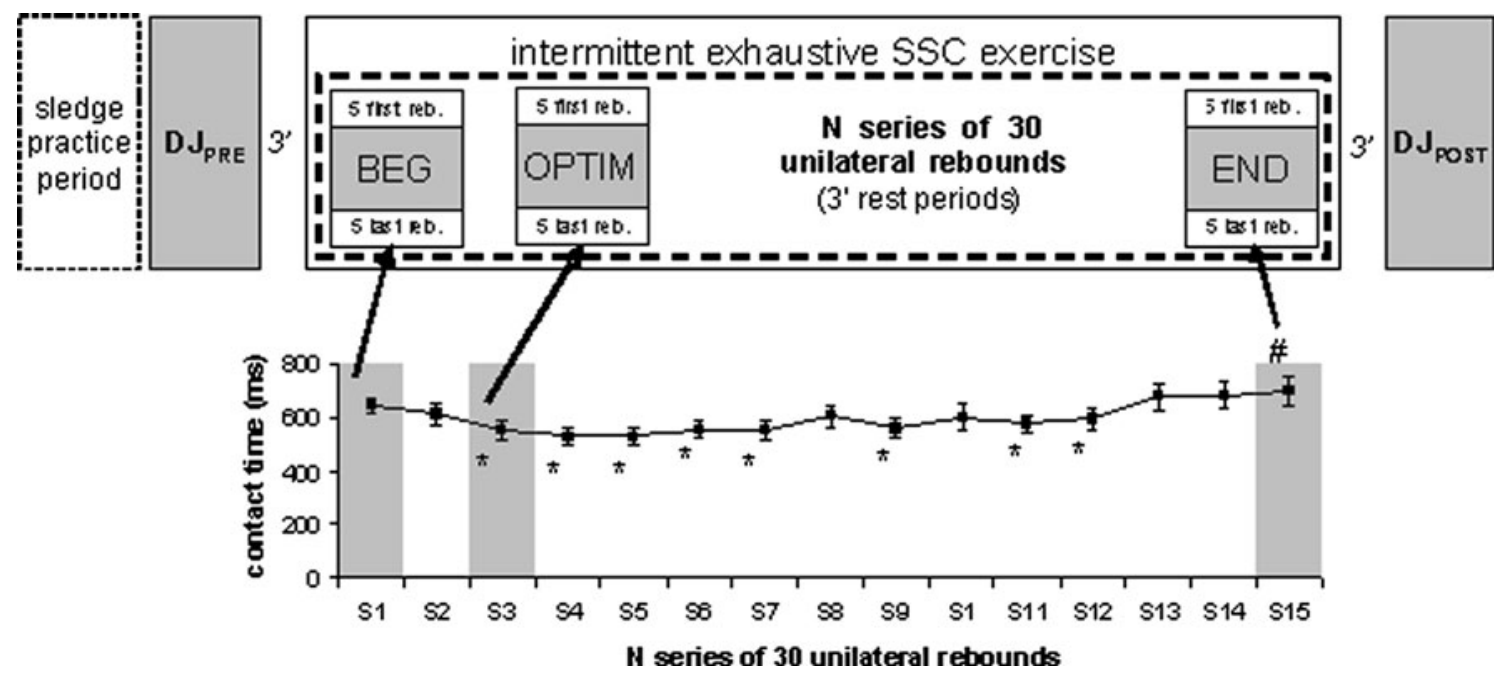

Fig. 1 The upper graph presents the overall (exercise and testing) protocol performed on a sledge apparatus. A warm-up and rebound practice preceded a maximal drop jump test performed 3 min before $\left(\mathrm{DJ}_{\mathrm{PRE}}\right)$ and after $\left(\mathrm{DJ}_{\mathrm{POST}}\right)$ an intermittent exhaustive SSC exercise. This exercise consisted of $N$ series of 30 unilateral rebounds repeated with 3 -min intermediate rest periods until exhaustion. The 6 th- 25 th rebounds of each series were kept for analysis, and averaged for the first (BEG), the optimized (OPTIM) and the last (END) series. As shown in the lower graph, the OPTIM series was individually determined as the first of at least two subsequent series with significantly shorter contact time than in the BEG (S1) series. $p<0.05$, significantly lower than $\mathrm{S} 1 .{ }^{\#} p<0.05$, significantly higher than $\mathrm{S} 1$ 
performance. Special attention was given to the respect of the $90^{\circ}$ knee bending. Exhaustion was considered as being attained when the subject could not reach the $70 \%$ rebound height during 15 successive rebounds of a given series (for details see Regueme et al. 2007).

\section{Maximal drop jump test}

To quantify the acute functional effects at exhaustion, a maximal DJ test was performed 3 min before $\left(D_{P R E}\right)$ and repeated 3 min after $\left(D_{\mathrm{POST}}\right)$ the exhaustive rebound exercise. Both $\mathrm{DJ}_{\mathrm{PRE}}$ and $\mathrm{DJ}_{\mathrm{POST}}$ tests included three unilateral maximal drop jumps, which were performed from the individually determined optimal dropping height with an intermediate 30-s time recovery. In case of a clear performance failure, an additional DJ was performed.

\section{Measurements}

The sledge displacement was recorded via an infinite potentiometer wheel attached to the sledge seat. The three components of the ground reaction force (GRF) were measured with a force plate (Kistler, 9281CA) set perpendicularly to the lower ends of the sledge rails. In order to quantify the shock transmitted to the lower limb, a 3D accelerometer ( $\pm 50 \mathrm{~g}$ Endevco, Isotron) recorded the tibial acceleration. The tibial accelerometer was strapped on the skin onto the anteromedial aspect of the lower leg $12 \mathrm{~cm}$ proximal to the medial malleolus (McMahon et al. 1987). Surface electromyographic (SEMG) activity was recorded from seven lower limb muscles: the soleus (SOL), gastrocnemii medialis (GAM) and lateralis (GAL), peroneus longus (PL), tibialis anterior (TA), vastii medialis (VM) and lateralis (VL) muscles. The positioning of the active surface electrodes (Contrôle Graphique Médical) followed the recommendations of SENIAM (1999). The SEMG signals were bandpass filtered $(10-500 \mathrm{~Hz})$ and amplified $(1,000 \times)$ using a specific recording system (Biopac, MP150). All these measurement devices were recorded at a 2,000-Hz sampling frequency and synchronised with LabView (National Instrument).

Data analysis

For the analysis of the exhaustive SSC exercise, each series of 30 rebounds was divided into three parts. The initial five rebounds (1st-5th) and the final five (26th-30th) were excluded from the analysis. The 6 th- 25 th rebounds were thus used as a representative sample of each series, from which every variable was then calculated using Matlab R2008b (The Mathworks).

The ground reaction force signals were first filtered by a critically damped low-pass filter (Robertson and Dowling
2003) with a 100-Hz cutting frequency. Each contact time (CT) was divided into two distinct phases: the braking phase as the descending sledge movement and the push-off phase as the ascending one. The reactive strength index (RSI) was calculated as the rebound height (m) divided by the contact time (s). The calculated force variables included peak amplitude and loading rate of the normal ground reaction force component during the braking phase and mean forces $\left(F_{\text {mean }}\right)$ for both braking and push-off phases. Lower limb stiffness was calculated during the braking phase by dividing the reaction force at the end of the braking phase by the corresponding sledge seat displacement (Kuitunen et al. 2007).

From the tibial acceleration signal the peak to peak amplitude (PPA) was calculated. In order to evaluate the severity of the shock transmitted through the tibia, the frequency content of the corresponding acceleration was studied from ground contact to $100 \mathrm{~ms}$ after. As the tibial impact acceleration signal is not stationary, the frequency content was investigated with a discrete wavelet analysis (von Tscharner 2000) using the Time-Frequency ToolBox for Matlab (Auger et al. 2009). The wavelet intensity (W) of the 10-100 Hz band frequency was calculated as the integral of the wavelet signal for the $0-100 \mathrm{~ms}$ time period. This frequency band is considered as representative of the tibial shock (Lafortune and Lake 1995). All acceleration variables were calculated for the medio-lateral (ML), antero-posterior (AP), proximo-distal (PD) components of the tibial acceleration.

Surface EMG signals were rectified and low-pass filtered with a $75-\mathrm{Hz}$ critically damped filter. Duration and average muscle activity (aEMG) were then calculated for the preactivation, braking and push-off phases. Timing of preactivation was determined for each rebound and muscle. The short-latency stretch-reflex component (referred to M1 according to Lee and Tatton 1982) was identified based on the original latencies proposed by Dietz et al. (1979) in running, once adjusted to the present condition of unilateral rebounds with the lower limbs on the sledge apparatus. As reported by Regueme et al. (2005a), the aEMG period was calculated as a $30-\mathrm{ms}$ window starting at $30 \mathrm{~ms}$ for the thigh muscles (VM, VL) (e.g. Horita et al. 1996) and at $40 \mathrm{~ms}$ for the leg muscles (SOL, GAM, GAM) (Dietz et al. 1979). For convenience, this time period was entitled M1, but it should be kept in mind that the obtained aEMG values included both supraspinal and stretch-reflex EMG components.

\section{Statistical analysis}

For the $\mathrm{DJ}_{\max }$ test, PRE-POST fatigue analysis used a bilateral pair-wised $t$ test to quantify exhaustion from the exerciseinduced changes in maximal rebound height. 
Fig. 2 Illustration with one subject's recordings of the BEG-OPTIM (left charts) and OPTIM-END (right charts) changes in ground reaction force (Fn), sledge displacement, proximo-distal tibial acceleration and rectified electromyographic activity of the soleus (SOL), gastrocnemius medialis (GAM), tibialis anterior (TA), peroneus longus (PL) and vastus medialis (VM) muscles. The OPTIM curves are presented in black, whereas the BEG and END curves are plotted in grey. Vertical dash lines represent the initial contact time and the transition from braking to push-off. From BEG to OPTIM, this subject presented a reduced TA preactivation and enhanced SOL and GAM M1 reflex response. From OPTIM to END larger changes took place, with increased braking and total contact times, reduced peak force and increased activity of SOL and TA during the push-off
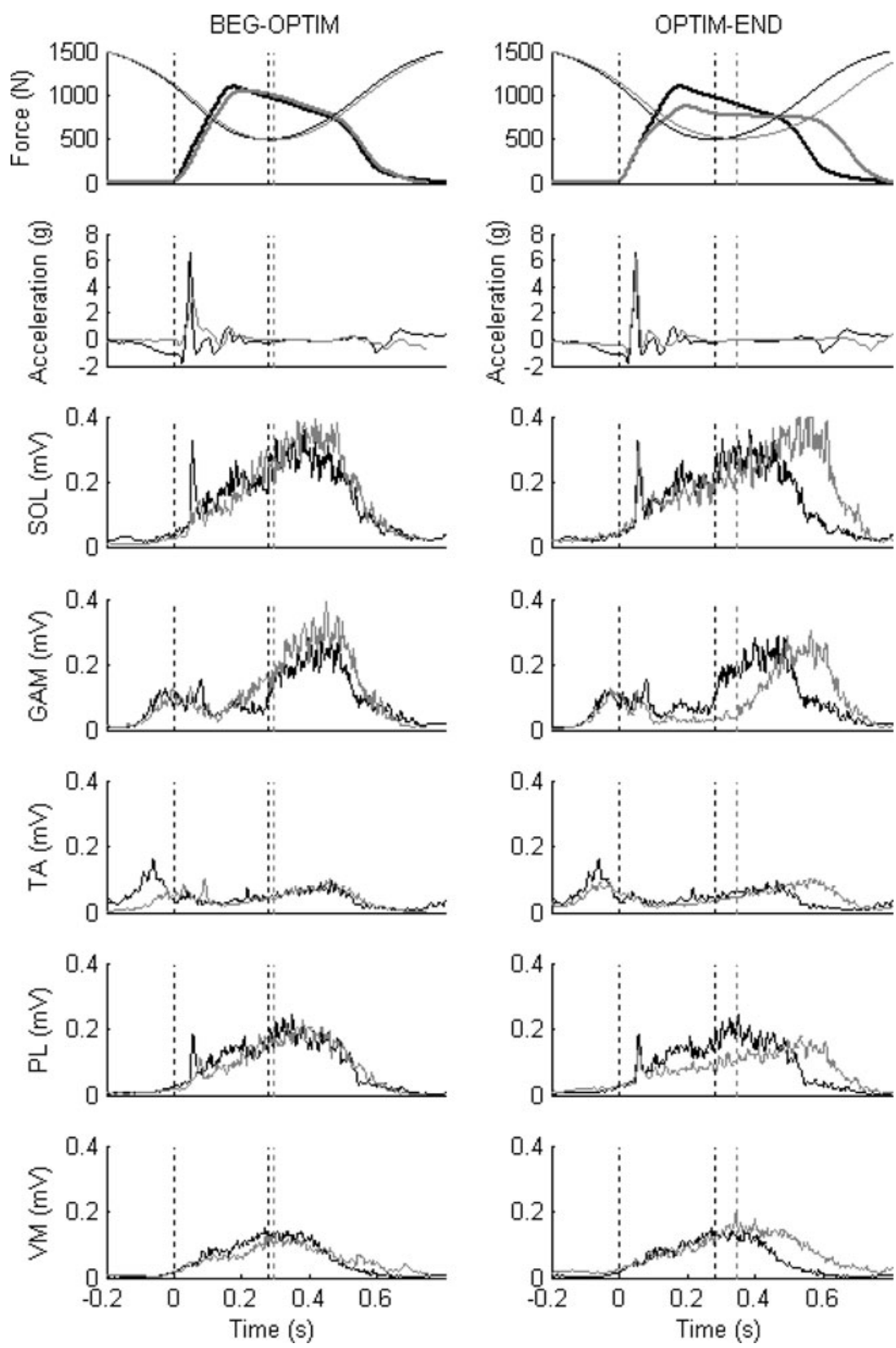

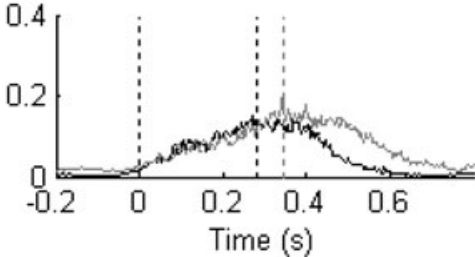

For the exhaustive rebound exercise, the first and last series of each subject were considered for convenience as BEG and END series. To allow the distinction between neuro-mechanical changes reflecting either SSC optimization or actual fatigue effects, a so-called optimized (OPTIM) series was statistically determined for each subject and used for BEG-OPTIM (optimization) and OPTIM-END (actual fatigue) $t$ test comparisons. As illustrated for one subject in the lower graph of Fig. 1, the individual OPTIM series was chosen as the first of at least two subsequent series that presented significantly shorter CT from the PRE series. The OPTIM series determination used one-way (testing time) analysis of variance (ANOVA) and
Tukey post-hoc test for inter-series comparison of the contact time (CT). As illustrated in Fig. 1, this allowed also the individual determination of the number of series stabilized at a short CT, before those showing the inverse trend towards exhaustion. The OPTIM series was also used as the $100 \%$ reference for the EMG analysis.

Intraclass correlation coefficients (Shrout and Fleiss 1979) model $\operatorname{ICC}_{(2, k)}$ were used to evaluate the similarity between the BEG-OPTIM and BEG-END changes. Correlation tests using a general linear model examined the possibility of relationships between time-dependant changes in the measured parameters as well as between muscle activities and mechanical variables. 


\section{Results}

On the average exhaustion was reached after $14 \pm 7.7$ series (6-32), which corresponded to a total of $420 \pm 230$ rebounds (180-960) and net exercise duration of $9.5 \pm 5 \mathrm{~min}$, with a mean duration of $40 \pm 3 \mathrm{~s}$ per series. The OPTIM-END comparison of the submaximal rebound height revealed a significant $7 \pm 7 \%$ reduction $(p=0.005)$, thus confirming that exhaustion had been reached. In this line, PRE-POST comparison of the DJ performance revealed a $10.3 \pm 11 \%$ decrease in maximal rebound height $(p=0.02)$. Figure 2 illustrates the normal ground reaction force, sledge displacement, PD tibial acceleration and EMG activity of the SOL, GAM, TA, PL and VM muscles for one subject.

SSC optimization in the submaximal rebound test

As expected, all subjects presented a clear decrease in CT associated with an increased reactive strength index (RSI) over the first few series of the exhaustive SSC exercise ( $p=0.01$ ). On the average, CT was stabilized at the $3 \pm 1$ series (considered as the OPTIM series) and remained as such for $8 \pm 5$ additional series before increasing again $3 \pm 2$ series before exhaustion.

Demonstrating an SSC optimization influence on the BEG-END changes, most of the significant BEG-OPTIM changes (Table 1) were positively correlated, with high intraclass correlation coefficients $\left(\mathrm{ICC}_{(2, k)}>0.89\right)$, to the global BEG-END ones. Only one of these parameters, PL aEMG activity during push-off, presented also a significant OPTIM-END change.
From BEG to OPTIM, CT decreased significantly by $13.7 \pm 11 \%(p=0.01)$ whereas total $F_{\text {mean }}$ increased by $10 \pm 9.3 \%(p=0.02)$ (Fig. 3). A positive relationship ( $p=0.001$ ) was found between the changes in SOL aEMG during the preactivation phase and the stretchreflex M1 period ( $r=0.92)$. The braking phase was characterised by reduced duration $(p=0.001)$ and sledge displacement $(p=0.002)$, with associated increases in $F_{\text {mean }}(p=0.005)$ and lower limb stiffness $(p=0.01)$. As shown in Fig. 4, the changes in lower limb stiffness were negatively correlated with the respective changes in total CT. The subsequent push-off phase was characterised by reduced duration $(p=0.007)$ and sledge displacement $(p=0.008)$, with an overall reduction in the average EMG activity (aEMG) of the GAM, GAL, PL and VM muscles $(p<0.05)$. The changes in SOL, GAL and PL aEMG activities were positively correlated with each other, both during the braking $(r>0.70 ; p<0.02)$ and the push-off $(r>0.88, p<0.001)$ phases.

Both BEG-OPTIM and BEG-END analysis revealed during the braking phase positive linear relationships $(p<0.02)$ between the changes in lower limb stiffness and aEMG activity of GAM ( $r=0.74$ and $r=0.87)$ and GAL ( $r=0.72$ and $r=0.76$ ) muscles. No relationship was found between lower limb stiffness and the EMG activity of other recorded muscles.

Actual fatigue effects in the submaximal rebound test

Only three of the significant BEG-END changes (Table 2) were confirmed by the OPTIM-END comparison and, thus, considered as actual fatigue effects. The

Table 1 Group mean $( \pm \mathrm{SD})$ values of the parameters recorded for the first (BEG), the optimized (OPTIM) and the last (END) rebound series

\begin{tabular}{|c|c|c|c|c|c|}
\hline SSC & Variables & BEG & OPTIM & END & $\mathrm{ICC}_{(2, k)}$ \\
\hline \multirow[t]{3}{*}{ Global } & $\mathrm{CT}(\mathrm{ms})$ & $662 \pm 83$ & $568 \pm 70 *$ & $569 \pm 95^{*}$ & 0.91 \\
\hline & $\mathrm{RSI}(\mathrm{m} / \mathrm{s})$ & $0.61 \pm 0.15$ & $0.73 \pm 0.19^{\#}$ & $0.70 \pm 0.26$ & - \\
\hline & Total $F_{\text {mean }}(\mathrm{bw})$ & $1.30 \pm 0.11$ & $1.44 \pm 0.18^{\#}$ & $1.42 \pm 0.23$ & - \\
\hline \multirow[t]{4}{*}{ Braking phase } & Phase duration (ms) & $315 \pm 44$ & $271 \pm 34^{*}$ & $268 \pm 45^{*}$ & 0.93 \\
\hline & Sledge displacement $(\mathrm{cm})$ & $51 \pm 8$ & $46 \pm 6^{*}$ & $44 \pm 6^{*}$ & 0.96 \\
\hline & $F_{\text {mean }}(\mathrm{bw})$ & $1.32 \pm 0.14$ & $1.45 \pm 0.17^{\#}$ & $1.48 \pm 0.24^{\#}$ & 0.91 \\
\hline & Lower limb stiffness (bw/m) & $1.21 \pm 0.31$ & $1.57 \pm 0.35^{\#}$ & $1.66 \pm 0.45^{\#}$ & 0.89 \\
\hline \multirow[t]{6}{*}{ Push-off phase } & Phase duration (ms) & $347 \pm 44$ & $297 \pm 41^{*}$ & $301 \pm 56^{*}$ & 0.89 \\
\hline & Sledge displacement $(\mathrm{cm})$ & $57 \pm 8$ & $52 \pm 6^{*}$ & $50 \pm 5^{*}$ & 0.92 \\
\hline & GAM aEMG (\%OPTIM) & $106 \pm 18$ & $100 *$ & $83 \pm 26^{*}$ & 0.90 \\
\hline & GAL aEMG (\%OPTIM) & $116 \pm 19$ & $100^{*}$ & $89 \pm 13^{*}$ & 0.94 \\
\hline & PL aEMG (\%OPTIM) & $120 \pm 31$ & $100 *$ & $81 \pm 15^{*, \S}$ & 0.95 \\
\hline & VM aEMG (\%OPTIM) & $136 \pm 48$ & $100 *$ & $98 \pm 16$ & - \\
\hline
\end{tabular}

Are presented only the parameters showing along the rebound exercise either significantly decreased $(* p<0.05)$ or increased $\left.{ }^{*} p<0.05\right)$ values as compared with BEG. Only one of these parameters presented also a significant OPTIM-END decrease $\left({ }^{\S} p<0.05\right)$. In case of both significant BEG-OPTIM and BEG-END changes, the corresponding intraclass correlation coefficients are presented in the last column 


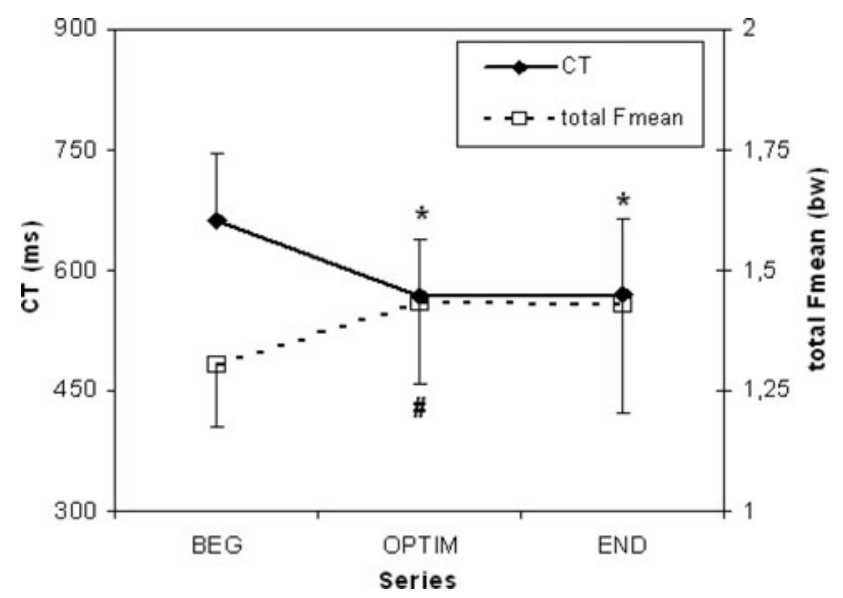

Fig. 3 Group averaged $( \pm \mathrm{SD})$ values of the total contact time $(\mathrm{CT})$ and mean normal force component (total $F_{\text {mean }}$ ) during the submaximal rebound exercise. From BEG to OPTIM, CT decreased significantly $(* p=0.01)$ whereas total $F_{\text {mean }}$ increased $\left({ }^{\#} p=0.02\right)$. No additional change was observed from OPTIM to END. The global BEG to END comparison revealed similar decreases $(* p=0.01)$ in $\mathrm{CT}$ than the PRE-OPTIM one

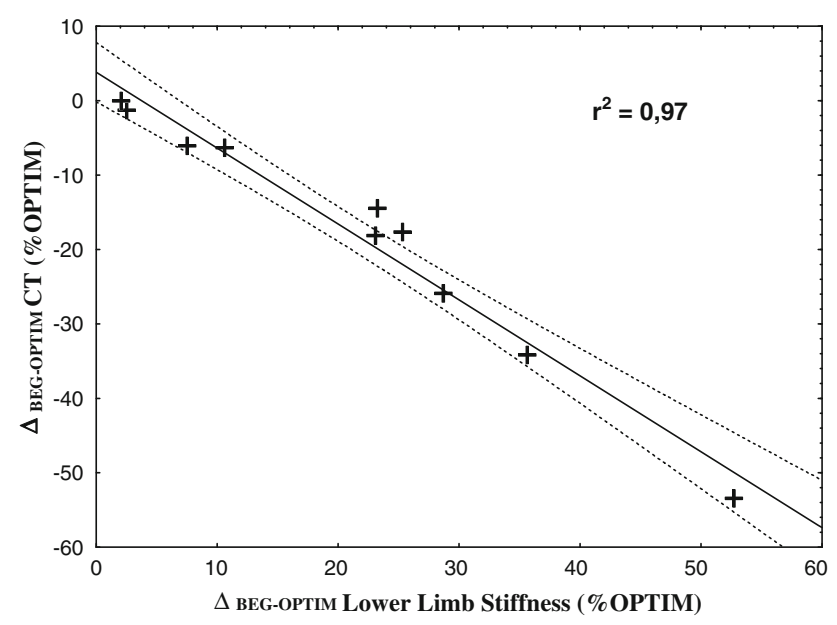

Fig. 4 Negative linear relationship between the BEG-OPTIM changes in lower limb stiffness and in contact time (CT). This indicated that an increase in lower limb stiffness was associated with a reduction in CT. The dash lines indicate the $95 \%$ CI

preactivation phase was characterised by an earlier PL muscle activation $(p=0.03)$. The braking phase presented an increased wavelet intensity of the medio-lateral (ML) tibial acceleration ( $p=0.03$ ) (Fig. 5) and both of these changes were negatively correlated with each other. The push-off phase showed reduced PL aEMG $(p=0.03)$.

The braking phase was again characterised by positive linear relationships $(p=0.02)$ between the lower limb stiffness and the aEMG activity of GAM $(r=0.78)$ and GAL $(r=0.71)$ muscles.
Isolated BEG-END changes during the submaximal rebound test

Isolated BEG-END changes were also observed that reflected progressive trends from BEG to END (Table 2). SOL preactivation started progressively earlier $(p=0.03)$. The braking phase presented significant increases in tibial acceleration variables: ML and AP peak-to-peak amplitudes (PPA), AP and PD wavelet intensities (W) $(\mathrm{p}<0.03)$. Reduced TA aEMG activity was observed during both braking and push-off phases $(p<0.04)$.

\section{Discussion}

This study examined the time course of neuro-mechanical changes along intermittent series of stretch-shortening cycles repeated until exhaustion on a sledge apparatus. The first hypothesis was that the expected decrease in contact time at the beginning of the SSC rebound exercise could reflect an improved SSC pattern rather than any early neuro-mechanical compensation for fatigue. In agreement with our earlier study (Regueme et al. 2005a), a clear CT reduction was observed in all subjects at the beginning of the submaximal rebound exercise. One critical aspect of the methodology previously used to determine the optimized rebound series was, however, the choice of the series performed at $25 \%$ of the individual total number of series. This implied that longer endurance performance would require an initially longer accommodation phase. As expected, the present study revealed the opposite trend and facilitated the use of an individualized determination of CT. Additional support for the use of CT in the detection of SSC optimization comes from the present BEG-OPTIM analysis, which revealed for the optimized series a braking phase of shorter duration, higher mean normal force value and smaller downward sledge displacement. As the submaximal rebound height remained constant, the early series were therefore characterised by a progressive increase in RSI. This is expected to reflect enhanced elastic energy storage during the braking phase and its subsequent recoil during the push-off phase (Kuitunen et al. 2007). In this line, a positive relationship was found in the present study between the PRE-OPTIM changes in lower limb stiffness and in RSI. Despite inter-individual variability in the neural strategies, the amplitude of the BEG-OPTIM changes in lower limb stiffness was mostly explained by the changes in GAM and GAL muscle activity. No significant change was found indeed in either SOL or TA muscle activity. In this line, biarticular muscles such as the gastrocnemii have been suggested to play a major role in the elastic energy storage and recoil (Sousa et al. 2007; Heise et al. 2008) as well as in the energy transfer between knee and ankle joints (van Ingen 
Table 2 Group mean $( \pm \mathrm{SD})$ values of the parameters recorded for the first (BEG), the optimized (OPTIM) and the last (END) rebound series

\begin{tabular}{|c|c|c|c|c|}
\hline SSC & Variables & BEG & OPTIM & END \\
\hline Performance & Rebound height $(\mathrm{cm})$ & $40 \pm 9$ & $41 \pm 9 *$ & $38 \pm 10$ \\
\hline \multirow[t]{2}{*}{ Preactivation phase } & SOL duration (ms) & $98 \pm 84^{\#}$ & $105 \pm 84$ & $117 \pm 81$ \\
\hline & PL duration (ms) & $102 \pm 80^{\#}$ & $107 \pm 77^{\#}$ & $123 \pm 81$ \\
\hline \multirow[t]{6}{*}{ Braking phase } & ML acc. peak to peak amplitude (g) & $10.2 \pm 5.1^{\#}$ & $11.3 \pm 5.8$ & $13.1 \pm 7$ \\
\hline & AP acc. peak to peak amplitude (g) & $10.7 \pm 3.9^{\#}$ & $11.8 \pm 4.8$ & $13.0 \pm 4.7$ \\
\hline & ML acc. total wavelet intensity $\left(\mathrm{g}^{2}\right)$ & $114 \pm 103^{\#}$ & $106 \pm 91^{\#}$ & $165 \pm 145$ \\
\hline & AP acc. total wavelet intensity $\left(\mathrm{g}^{2}\right)$ & $112 \pm 108^{\#}$ & $132 \pm 116$ & $155 \pm 119$ \\
\hline & PD acc. total wavelet intensity ( $\mathrm{g}^{2}$ ) & $94 \pm 47^{\#}$ & $106 \pm 44$ & $124 \pm 66$ \\
\hline & TA aEMG (\%OPTIM) & $110 \pm 32 *$ & 100 & $83 \pm 16$ \\
\hline \multirow[t]{3}{*}{ Push-off phase } & PL aEMG (\%OPTIM) & $120 \pm 31^{*, \S}$ & $100 *$ & $81 \pm 15$ \\
\hline & TA aEMG (\%OPTIM) & $107 \pm 8^{*}$ & 100 & $92 \pm 14$ \\
\hline & VL aEMG (\%OPTIM) & $128 \pm 66$ & $100 *$ & $87 \pm 14$ \\
\hline
\end{tabular}

Only the parameters showing significantly higher $(* p<0.05)$ or lower $\left({ }^{\#} p<0.05\right)$ values in the early (BEG or OPTIM) series than at exhaustion (END) are presented. One parameter showed also a significant BEG-OPTIM (§) decrease. Medio-lateral, antero-posterior and proximo-distal acceleration components are referred as ML, AP and PD Acc
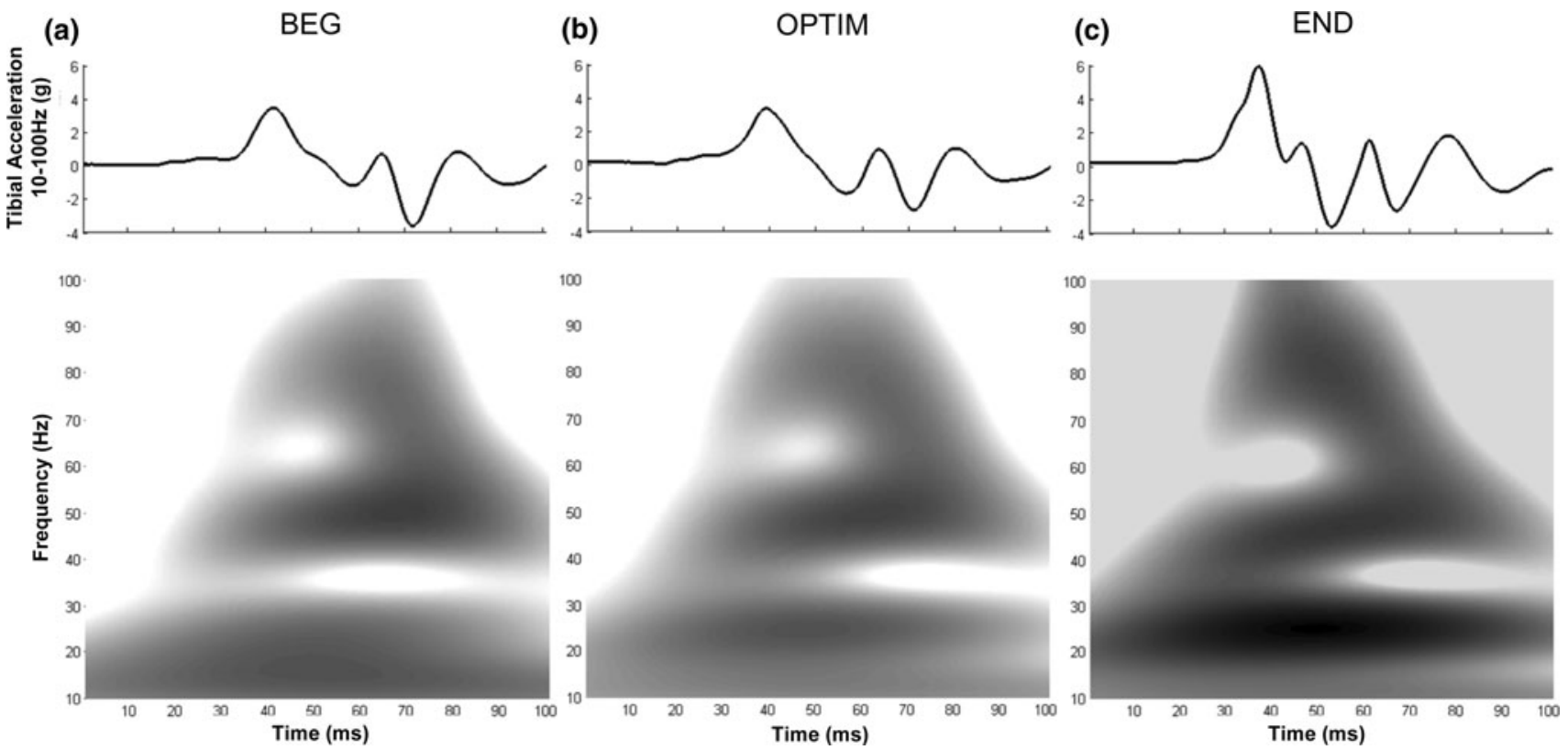

Fig. 5 ML tibial acceleration (up) and wavelet spectrum (down) of the averaged 6th-25th rebounds in the BEG (a), OPTIM (b) and END (c) series of a representative subject in the submaximal rebound test. All charts represent the tibial acceleration in the $10-$ to $100-\mathrm{Hz}$

Schenau et al. 1987). Emphasizing the role of central neural adjustments, SOL aEMG changes during the stretch-reflex M1 period were positively correlated with its changes in preactivation. Although such a relationship might be partly attributed to the supraspinal voluntary drive included in the present calculation of M1, only one of our previous studies reported a similar finding (Regueme et al. 2005b). In the other studies (Regueme et al. 2005a, 2007), no relationship was observed and different trends of changes were found during the M1 period and its preceding $30 \mathrm{~ms}$ period frequency band, from the initial ground contact to $100 \mathrm{~ms}$ later. In the lower charts, the grey scale of the wavelet spectrum goes from white (low intensities) to black (high intensities)

known as purely centrally regulated. Finally, the push-off phase was characterised by an overall reduction in the gastrocnemii and vastus medialis muscle activation that can be considered as additional confirmation of a clear SSC optimization (Kyröläinen and Komi 1995b, Paavolainen et al. 1999). Additional support comes from the fact that half of the significant BEG-END changes in the submaximal rebound test were highly correlated and, thus, mostly explained by the early BEG-OPTIM changes. These overall mechanical and EMG changes give support to our first 
hypothesis that the large CT reduction repeatedly reported at the beginning of exhaustive rebound exercises on the sledge could reflect mostly SSC optimization. This is confirming our expectation that erroneous conclusions might have been drawn on SSC fatigue effects by using the beginning of the rebound exercise as a reference.

When dealing with the actual fatigue and exhaustion effects, one may question the fact that the OPTIM-END changes remained quite limited for an SSC exercise expected to lead to exhaustion. In particular, the lower limb stiffness was found to stay unchanged from OPTIM to END and no significant change was found either in the recorded muscle activity. As recently reviewed by Nicol et al. (2006), the time to exhaustion varies a lot among reported protocols and subjects, being clearly dependent on the exercise mode (continuous or intermittent) and intensity as well as on the subjects' training level. The present reductions of 7 and 10\% found in submaximal and maximal (DJ) rebound heights after the intermittent rebound exercise are close to the earlier results of Regueme et al. (2005a, b, 2007) when using similar protocols. Emphasizing also the existence of a large inter-individual variability in the strategies and capacity to compensate for fatigue, the SSC pattern analysis (based on the CT parameter) revealed a stabilized pattern that lasted from 3 to 13 rebound series depending on the subjects. Similarly, its subsequent deterioration lasted 1-5 series prior to resulting in a large drop in rebound performance and, thus, in the final stop of the exercise. In this line, although the OPTIM-END comparison confirmed the BEG-OPTIM observation of the major role played by the gastrocnemii muscles in the lower limb stiffness regulation, a large inter-individual variability was found again in the changes of both parameters. This was further confirmed by the absence of clear relationship between the BEG-OPTIM and the OPTIM-END changes. Similarly, conflicting results have been reported in the SSC literature. At the end of severe continuous running or jumping exercises, some studies (Dutto and Smith 2002; Kuitunen et al. 2007) reported a significant reduction in leg stiffness whereas no change was found by Avogadro et al. (2003). On the other hand, no change was reported either during (Morin et al. 2006) or across (Morin et al. 2006; Girard et al. 2011) fatiguing sprints trials. While hopping at a constant frequency, Bonnard et al. (1994) found shorter CT and greater vertical peak force that would suggest an increase of lower limb stiffness with fatigue. Part of these discrepancies may be attributed to the way a given task may either favour or limit the intervention of inter-muscular neural compensations. The submaximal rebound task on the sledge used in the present exhaustive SSC protocol has already been demonstrated by Horita (2000) and Regueme et al. (2005a) as favouring inter- and intra-muscle group compensations. Despite the observation of limited actual fatigue effects it is suggested that the present intermittent rebound protocol did lead the subject to exhaustion.

Additional support comes from the confirmation of our expectation that the severity of the shock transmitted through the tibia would increase towards exhaustion. The wavelet intensity of the ML tibial acceleration was indeed found to increase, suggesting that the tibia endured more transverse shock at the end of the exhaustive exercise than at the beginning. Although tibial acceleration was early explored in three dimensions by Lafortune (1991), it is unfortunate that only the proximo-distal (PD) axis does remain of interest in the recent literature. Interestingly, however, Moran and Marshall (2006) reported after exhaustive running an increased PD tibial acceleration when tested in maximal DJ from a low $(30 \mathrm{~cm})$ dropping height. Moreover, both amplitude and median frequency of the PD tibial acceleration have been found to increase along prolonged running exercises (Mizrahi et al. 2000; Verbitsky et al. 1998). As proposed by Mizrahi et al. (2000) one mechanical consequence of such increase in tibial acceleration during prolonged exercise might result in the development of excessive tibial bending stresses and higher risk of stress injury. It is assumed that the present increase of the ML tibial acceleration intensity constituted also an injury risk factor. It is noteworthy that the subjects who presented limited ML instability were those who activated earlier their PL muscle in the preactivation phase. Emphasizing the potential role of PL in the loss of ML stability, all the subjects presented a reduced PL activity during the push-off phase. Similar observation has been reported during a fatiguing walk (Gefen 2002) with associated lateral shift of the centre of pressure. This deterioration in foot stability was suggested to favour the occurrence of ankle sprains and to contribute to the observed increase in metatarsal stresses. Thus, limited SSC fatigue effects are expected to allow neuro-mechanical adjustments to occur at the expense of deleterious impairments in the ML instability of the foot or impact shock transmitted to the leg.

\section{Conclusion}

This study intended to improve the testing of the neuromechanical changes induced by exhaustive rebound exercise. Our results give support to the use of the contact time parameter to follow the initial SSC optimization phase in order to determine individually the pre-fatigue (optimized) SSC reference. They demonstrate clearly that potential erroneous interpretation might be drawn when using the early beginning of this type of exercise instead. The present methodology would need to be confirmed, however, in other SSC testing conditions than in a rebound task on the 
sledge apparatus. When dealing with the potential risks of overuse injury in exhaustive rebound exercise, ML tibial acceleration appears of particular relevance, even in the case of limited functional effects.

Acknowledgments Cédric Morio was supported by a grant of the French National Agency of Technological Research (ANRT), no. 2007/1067. The authors would also like to thank Dr. Guillaume Rao for his helpful comments on wavelet analysis.

\section{References}

Auger F, Flandrin P, Gonçalvès P, Lemoine O (2009) Time-Frequency Toolbox reference guide. Centre National de la Recherche Scientifique

Avogadro P, Dolenec A, Belli A (2003) Changes in mechanical work during severe exhausting running. Eur J Appl Physiol 90(1-2):165-170

Bigland-Ritchie B, Woods JJ (1984) Changes in muscle contractile properties and neural control during human muscular fatigue. Muscle Nerve 7(9):691-699

Bonnard M, Sirin AV, Oddson L, Thorstensson A (1994) Different strategies to compensate for the effects of fatigue revealed by neuromuscular adaptation processes in humans. Neurosci Lett 166:101-105

Candau R, Belli A, Millet GY, Georges D, Barbier B, Rouillon JD (1998) Energy cost and running mechanics during a treadmill run to voluntary exhaustion in humans. Eur J Appl Physiol Occup Physiol 77(6):479-485

Clark RA (2009) The effect of training status on inter-limb joint stiffness regulation during repeated maximal sprints. J Sci Med Sport $12: 406-410$

Cormie P, McGuigan MR, Newton RU (2010) Changes in the eccentric phase contribute to improved stretch-shorten cycle performance after training. Med Sci Sports Exerc 42(9):1731-1744

Dalleau G, Belli A, Bourdin M, Lacour JR (1998) The spring-mass model and the energy cost of treadmill running. Eur J Appl Physiol 77:257-263

Dietz V, Schmidtbleicher D, Noth J (1979) Neuronal mechanisms of human locomotion. J Neurophysiol 42(5):1212-1222

Dutto DJ, Smith GA (2002) Changes in spring-mass characteristics during treadmill running to exhaustion. Med Sci Sports Exerc 34:1324-1331

Enoka RM, Duchateau J (2008) Muscle fatigue: what, why and how it influences muscle function. J Physiol 586:11-23

Finni T, Kyröläinen H, Avela J, Komi PV (2003) Maximal but not submaximal performance is reduced by constant-speed $10-\mathrm{km}$ run. J Sports Med Phys Fitness 43(3):411-717

Flanagan EP, Comyns TM (2008) The use of contact time and the reactive strength index to optimize fast stretch-shortening cycle training. Strength Cond J 30(5):32-38

Gefen A (2002) Biomechanical analysis of fatigued-related foot injury mechanisms in athletes and recruits during intensive marching. Med Biol Eng Comput 40:302-310

Girard O, Micallef JP, Millet GP (2011) Changes in spring-mass model characteristics during repeated running sprints. Eur J Appl Physiol 111(1):125-134

Gollhofer A, Komi PV, Miyashita M, Aura O (1987) Fatigue during stretch-shortening cycle exercises: changes in mechanical performance of human skeletal muscle. Int J Sports Med 8(2):71-78

Hardin EC, Van den Bogert AJ, Hamill J (2004) Kinematic adaptations during running: effects of footwear, surface, and duration. Med Sci Sports Exerc 36(5):838-844
Hasegawa H, Yamauchi T, Kraemer WJ (2007) Foot strike patterns of runners at the $15-\mathrm{km}$ point during an elite-level half marathon. J Strength Cond Res 21(3):888-893

Heise G, Shinohara M, Binks L (2008) Biarticular leg muscles and links to running economy. Int J Sports Med 29(8):688-691

Horita T (2000) Stiffness regulation during stretch-shortening cycle exercise. Department of Biology of Physical Activity. Research series published by the University of Jyväskylä, Finland

Horita T, Komi PV, Nicol C, Kyröläinen H (1996) Stretch shortening cycle fatigue: interactions among joint stiffness, reflex, and muscle mechanical performance in the drop jump. Eur J Appl Physiol Occup Physiol 73(5):393-403

Komi PV (2000) Stretch-shortening cycle: a powerful model to study normal and fatigued muscle. J Biomech 33(10):1197-1206

Komi PV, Gollhofer A (1997) Stretch reflexes can have an important role in force enhancement during ssc exercise. J Appl Biomech $13: 451-460$

Komi PV, Nicol C (2010) Stretch-shortening cycle of muscle function. In: Komi PV (ed) Neuromuscular aspects of sport performance, 1st edn. Wiley-Blackwell, Chichester, pp 15-31

Kuitunen S, Kyroläinen H, Avela J, Komi PV (2007) Leg stiffness modulation during exhaustive stretch-shortening cycle exercise. Scand J Med Sci Sports 17:67-75

Kyröläinen H, Komi PV (1995a) Differences in mechanical efficiency between power- and endurance-trained athletes while jumping. Eur J Appl Physiol Occup Physiol 70(1):36-44

Kyröläinen H, Komi PV (1995b) The function of neuromuscular system in maximal stretch-shortening cycle exercises: comparison between power- and endurance-trained athletes. J Electromyogr Kinesiol 5:15-25

Lafortune MA (1991) Three-dimensional acceleration of the tibia during walking and running. J Biomech 24:877-886

Lafortune MA, Lake MJ (1995) Human pendulum approach to simulate and quantify locomotor impact loading. J Biomech 28(9):1111-1114

Lee RG, Tatton WG (1982) Long latency reflexes to imposed displacements of the human wrist: dependence on duration of movement. Exp Brain Res 45:207-216

Martin V, Kerhervé H, Messonnier LA, Banfi JC, Geyssant A, Bonnefoy R, Féasson L, Millet GY (2010) Central and peripheral contributions to neuromuscular fatigue induced by a $24-\mathrm{h}$ treadmill run. J Appl Physiol 108(5):1224-1233

McMahon TA, Valiant G, Frederick EC (1987) Groucho running. J Appl Physiol 62(6):2326-2337

Mizrahi J, Verbitsky O, Isakov E (2000) Fatigue-related loading imbalance on the shank in running: a possible factor in stress fractures. Ann Biomed Eng 28:463-469

Moran KA, Marshall BM (2006) Effect of fatigue on tibial impact accelerations and knee kinematics in drop jumps. Med Sci Sports Exerc 38:1836-1842

Morin JB, Jeannin T, Chevallier B, Belli A (2006) Spring-mass model characteristics during sprint running: correlation with performance and fatigue-induced changes. Int J Sports Med 27:158-165

Morin JB, Samozino P, Peyrot N (2009) Running pattern changes depending on the level of subjects' awareness of the measurements performed: a "sampling effect" in human locomotion experiments? Gait Posture 30(4):507-510

Nicol C, Komi PV (2010) Stretch-shortening cycle fatigue. In: Komi PV (ed) Neuromuscular aspects of sport performance, 1st edn. Wiley-Blackwell, Chichester, pp 183-215

Nicol C, Komi PV, Marconnet P (1991) Fatigue effects of marathon running on neuromuscular performance. I. Changes in muscle force and stiffness characteristics. Scand J Med Sci Sports 1:10-17

Nicol C, Komi PV, Horita T, Kyröläinen H, Takala TE (1996) Reduced stretch-reflex sensitivity after exhausting stretch-shortening cycle exercise. Eur J Appl Physiol Occup Physiol 72:401-409 
Nicol C, Avela J, Komi PV (2006) The stretch-shortening cycle: a model to study naturally occurring neuromuscular fatigue. Sports Med 36:977-999

Nummela A, Keränen T, Mikkelsson LO (2007) Factors related to top running speed and economy. Int J Sports Med 28(8):655-661

Nummela AT, Heath KA, Paavolainen LM, Lambert MI, Gibson ASC, Rusko HK, Noakes TD (2008) Fatigue during a 5-km running time trial. Int J Sports Med 29:738-745

Paavolainen L, Nummela A, Rusko H, Häkkinen K (1999) Neuromuscular characteristics and fatigue during $10 \mathrm{~km}$ running. Int $\mathrm{J}$ Sports Med 20(8):516-521

Regueme SC, Nicol C, Barthèlemy J, Grélot L (2005a) Acute and delayed neuromuscular adjustments of the triceps surae muscle group to exhaustive stretch-shortening cycle fatigue. Eur J Appl Physiol 93:398-410

Regueme SC, Barthèlemy J, Gauthier GM, Nicol C (2005b) Central nervous system contralateral adjustments after unilateral muscle fatigue. In: Gantchev (ed) Basic motor control to functional recovery, vol IV. Marin Drinov Academic Publishing House, Sofia, pp 300-307

Regueme SC, Barthèlemy J, Nicol C (2007) Exhaustive stretchshortening cycle exercise: no contralateral effects on muscle activity in maximal motor performances. Scand J Med Sci Sports 17:547-555

Robertson DGE, Dowling JJ (2003) Design and responses of Butterworth and critically damped digital filters. J Electromyogr Kinesiol 13:569-573

Shrout PE, Fleiss JL (1979) Intraclass correlations: uses in assessing rater reliability. Psychol Bull 86(2):420-428

Sousa F, Ishikawa M, Vilas-Boas JP, Komi PV (2007) Intensity- and muscle-specific fascicle behaviour during human drop jumps. J Appl Physiol 102:382-389

Spurrs RW, Murphy AJ, Watsford ML (2003) The effect of plyometric training on distance running performance. Eur J Appl Physiol 89(1):1-7

van Ingen Schenau GJ, Bobbert MF, Rozendal RH (1987) The unique action of bi-articular muscles in complex movements. J Anat 155:1-5

Verbitsky O, Mizrahi J, Voloshin A, Treiger J, Isakov E (1998) Shock transmission and fatigue in human running. J Appl Biomech 14:300-311

von Tscharner V (2000) Intensity analysis in time-frequency space of surface myoelectric signals by wavelets of specified resolution. J Electromyogr Kinesiol 10:433-445 